\title{
Proportional Derivative Control with Inverse Dead-Zone for Pendulum Systems
}

\author{
José de Jesús Rubio, Zizilia Zamudio, Jaime Pacheco, and Dante Mújica Vargas \\ Sección de Estudios de Posgrado e Investigación, ESIME Azcapotzalco, Instituto Politécnico Nacional, Avenida de las Granjas No. 682, \\ Colonia Santa Catarina, 02250 México, DF, Mexico \\ Correspondence should be addressed to José de Jesús Rubio; jrubioa@ipn.mx
}

Received 11 July 2013; Revised 30 August 2013; Accepted 2 September 2013

Academic Editor: Zidong Wang

Copyright (C) 2013 José de Jesús Rubio et al. This is an open access article distributed under the Creative Commons Attribution License, which permits unrestricted use, distribution, and reproduction in any medium, provided the original work is properly cited.

A proportional derivative controller with inverse dead-zone is proposed for the control of pendulum systems. The proposed method has the characteristic that the inverse dead-zone is cancelled with the pendulum dead-zone. Asymptotic stability of the proposed technique is guaranteed by the Lyapunov analysis. Simulations of two pendulum systems show the effectiveness of the proposed technique.

\section{Introduction}

Nonsmooth nonlinear characteristics such as dead-zone, backlash, and hysteresis are common in actuators, sensors such as mechanical connections, hydraulic servovalves, and electric servomotors; they also appear in biomedical systems. Dead-zone is one of the most important nonsmooth nonlinearities in many industrial processes, which can severely limit the system performance, and its study has been drawing much interest in the control community for a long time [1].

There is some research about control systems. In [2], the stabilization of the inverted-car pendulum is presented. The stabilization of the Furuta pendulum is introduced in [3]. In [4], the dissipative control problem is investigated for a class of discrete time-varying systems. The distributed $H_{\infty}$ filtering problem for a class of nonlinear systems is considered in [5]. The recursive finite-horizon filtering problem for a class of nonlinear time-varying systems is addressed in $[6,7]$. In [8], the authors present a solution to the problem of the quadratic mini-max regulator for polynomial uncertain systems. The problem of a two-player differential game affected by matched uncertainties with only the output measurement available for each player is considered by [9]. The stability analysis and $H_{\infty}$ control for a class of discrete time switched linear parameter-varying systems are concerned in [10]. The sliding mode control problem for uncertain nonlinear discretetime stochastic systems with $\mathrm{H}_{2}$ performance constraints is designed in [11]. In [12], the sliding mode control problem is considered for discrete-time systems. In [13], the description about the modelling and control of wind turbine system is addressed. A model to describe the dynamics of a homogeneous viscous fluid in an open pipe is introduced in [14]. In [15], the authors consider the problems of robust stability and $H_{\infty}$ control for a class of networked control systems with long-time delays. The $H_{\infty}$ control issue for a class of networked control systems with packet dropouts and timevarying delays is introduced in [16]. From the above studies, in $[2,3,8,9,13,14]$, the authors propose proportional derivative controls; however, none considers systems with dead-zone inputs.

There is some work about the control of systems with dead-zone inputs. In [17-22], the authors proposed the control of nonlinear systems with dead-zone inputs. Nevertheless, they do not research about the pendulum systems. The pendulum dynamic models have different structures with respect to the nonlinear systems addressed in the above papers; thus, a new design may be developed.

In this paper, a proportional derivative controller with inverse dead-zone is proposed for the control of pendulum systems with dead-zone inputs. One main contribution of this study is that the pendulum dynamic model is rewritten as 
a robotic dynamic model to satisfy a property, and later, the property is applied to guarantee the stability of the proposed controller.

The paper is organized as follows. In Section 2, the dynamic model of the robotic arm with dead-zone inputs is presented. In Section 3, the dynamic model of the pendulum systems with dead-zone inputs is presented. In Section 4, the proportional derivative controller with inverse dead-zone is introduced. In Section 5, the proposed method is used for the regulation of two pendulum systems. Section 6 presents conclusions and suggests future research directions.

\section{Dynamic Model of the Robotic Arms with Dead-Zone Inputs}

The main concern of this section is to understand some concepts of robot dynamics. The equation of motion for the constrained robotic manipulator with $n$ degrees of freedom, considering the contact force and the constraints, is given in the joint space as follows:

$$
M(q) \ddot{q}+C(q, \dot{q}) \dot{q}+G(q)=\tau,
$$

where $q \in \mathfrak{R}^{n \times 1}$ denotes the joint angles or link displacements of the manipulator, $M(q) \in \mathfrak{R}^{n \times n}$ is the robot inertia matrix which is symmetric and positive definite, $C(q, \dot{q}) \in \Re^{n \times n}$ contains the centripetal and Coriolis terms and $G(q)$ are the gravity terms, and $\tau$ denotes the dead-zone output. The nonsymmetric dead-zone can be represented by

$$
\tau=\mathrm{DZ}(v)= \begin{cases}m_{r}\left(v-b_{r}\right) & v \geq b_{r} \\ 0 & b_{l}<v<b_{r} \\ m_{l}\left(v-b_{l}\right) & v \leq b_{l},\end{cases}
$$

where $m_{r}$ and $m_{l}$ are the right and left constant slopes for the dead-zone characteristic and $b_{r}$ and $b_{l}$ represent the right and left breakpoints. Note that $v$ is the input of the dead-zone and the control input of the global system.

Define the following two states as follows:

$$
\begin{aligned}
& x_{1}=q \in \mathfrak{R}^{n \times 1}, \\
& x_{2}=\dot{q} \in \mathfrak{R}^{n \times 1}, \\
& u=\tau \in \mathfrak{R}^{n \times 1},
\end{aligned}
$$

where $x_{1}=\left[\begin{array}{ll}x_{11} & x_{12}\end{array}\right]^{T}=\left[\begin{array}{ll}q_{1} & q_{2}\end{array}\right]^{T}, x_{2}=\left[\begin{array}{ll}x_{21} & x_{22}\end{array}\right]^{T}=$ $\left[\begin{array}{ll}\dot{q}_{1} & \dot{q}_{2}\end{array}\right]^{T}$ for $n=2$. Then (1) can be rewritten as

$$
\begin{gathered}
\dot{x}_{1}=x_{2}, \\
M\left(x_{1}\right) \dot{x}_{2}+C\left(x_{1}, x_{2}\right) x_{2}+G\left(x_{1}\right)=u
\end{gathered}
$$

where $M\left(x_{1}\right), C\left(x_{1}, x_{2}\right)$, and $G\left(x_{1}\right)$ are described in (1), the dead-zone $u$ is $[17,19,20,22]$

$$
u=\mathrm{DZ}(v)= \begin{cases}m_{r}\left(v-b_{r}\right) & v \geq b_{r} \\ 0 & b_{l}<v<b_{r} \\ m_{l}\left(v-b_{l}\right) & v \leq b_{l},\end{cases}
$$

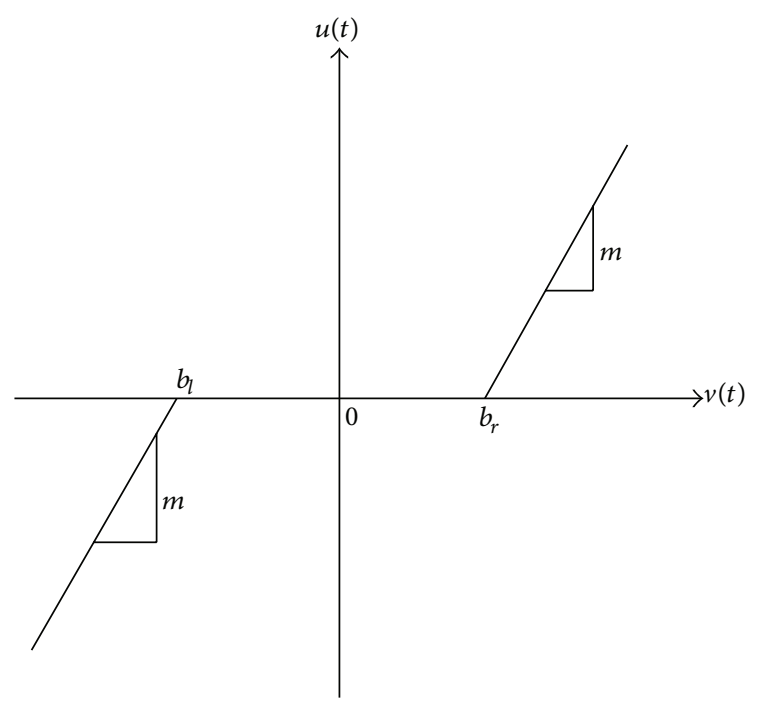

Figure 1: The dead-zone.

the parameters $m_{r}, m_{l}, b_{r}$, and $b_{l}$ are described in (2), and $v$ is the control input of the system. Figure 1 shows the dead-zone [17].

Property 1. The inertia matrix is symmetric and positive definite; that is, [23-25]

$$
m_{1}|x|^{2} \leq x^{T} M\left(x_{1}\right) x \leq m_{2}|x|^{2}
$$

where $m_{1}, m_{2}$ are known positive scalar constants; $x=$ $\left[x_{1}, x_{2}\right]^{T}$.

Property 2. The centripetal and Coriolis matrix is skewsymmetric, that is, satisfies the following relationship [2325]:

$$
x^{T}\left[\dot{M}\left(x_{1}\right)-2 C\left(x_{1}, x_{2}\right)\right] x=0,
$$

where $x=\left[x_{1}, x_{2}\right]^{T}$.

The normal proportional derivative controller is

$$
u=-K_{p} \tilde{x}_{1}-K_{d} \tilde{x}_{2},
$$

where $\tilde{x}_{1}=x_{1}-x_{1}^{d}$ and $\tilde{x}_{2}=x_{2}-x_{2}^{d}$ and $K_{p}$ and $K_{d}$ are positive definite, symmetric, and constant matrices.

\section{Dynamic Model of the Pendulum Systems with Dead-Zone Inputs}

The dynamic model of the pendulum systems can be rewritten as the dynamic model of the robotic arms; however Property 2 is not directly satisfied. Pendulum dynamic models are rewritten as the robotic dynamic models because in this study, if the above sentence is true, Property 2 of the robotic systems can be used to guarantee the stability of the controller applied to the pendulum systems. The following lemmas let to modify the Property 2 for its application in the pendulum systems. 
Lemma 1. A pendulum model can be rewritten as a robotic arm model (4). Nevertheless, it cannot satisfy Property 2.

Proof. Consider $n=2$ for the pendulum systems in (4); it gives

$$
\begin{gathered}
\dot{x}_{1}=x_{2}, \\
M\left(x_{1}\right) \dot{x}_{2}+C\left(x_{1}, x_{2}\right) x_{2}+G\left(x_{1}\right)=u,
\end{gathered}
$$

where

$$
\begin{gathered}
M\left(x_{1}\right)=\left[\begin{array}{ll}
m_{11} & m_{12} \\
m_{21} & m_{22}
\end{array}\right], \\
C\left(x_{1}, x_{2}\right)=\left[\begin{array}{ll}
c_{11} & c_{12} \\
c_{21} & c_{22}
\end{array}\right], \\
G\left(x_{1}\right)=\left[\begin{array}{l}
g_{1} \\
g_{2}
\end{array}\right]
\end{gathered}
$$

and $M\left(x_{1}\right), C\left(x_{1}, x_{2}\right)$, and $G\left(x_{1}\right)$ are selected from the pendulum dynamic model, and $x_{1}$ and $x_{2}$ are defined in (3). Consequently,

$$
x^{T}\left[\dot{M}\left(x_{1}\right)-2 C\left(x_{1}, x_{2}\right)\right] x \neq 0 .
$$

Lemma 2. Pendulum model (4) can be rewritten as follows:

$$
\begin{gathered}
\dot{x}_{1}=x_{2}, \\
M\left(x_{1}\right) \dot{x}_{2}=-\widehat{C}\left(x_{1}, x_{2}\right) x_{2}-N\left(x_{1}, x_{2}\right)+u
\end{gathered}
$$

where

$$
\begin{gathered}
M\left(x_{1}\right)=\left[\begin{array}{ll}
m_{11} & m_{12} \\
m_{21} & m_{22}
\end{array}\right], \\
\widehat{C}\left(x_{1}, x_{2}\right)=\left[\begin{array}{ll}
\widehat{c}_{11} & \widehat{c}_{12} \\
\widehat{c}_{21} & \widehat{c}_{22}
\end{array}\right], \\
N\left(x_{1}, x_{2}\right)=\left[\begin{array}{l}
n_{1} \\
n_{2}
\end{array}\right]
\end{gathered}
$$

the input $u$ is given by (5), $c_{11}=\widehat{c}_{11}+\bar{c}_{11}, c_{12}=\widehat{c}_{12}+\bar{c}_{12}$, $c_{21}=\widehat{c}_{21}+\bar{c}_{21}, c_{22}=\widehat{c}_{22}+\bar{c}_{22}, n_{1}=g_{1}+\bar{c}_{11} x_{21}+\bar{c}_{12} x_{22}$, $n_{2}=g_{2}+\bar{c}_{21} x_{21}+\bar{c}_{22} x_{22}, x_{1}$ and $x_{2}$ are defined in (3), and the following modified property is satisfied:

$$
x^{T}\left[\dot{M}\left(x_{1}\right)-2 \widehat{C}\left(x_{1}, x_{2}\right)\right] x=0 .
$$

Proof. Consider $n=2$ for the pendulum systems in (4); it gives

$$
\begin{aligned}
& M\left(x_{1}\right) \dot{x}_{2}+C\left(x_{1}, x_{2}\right) x_{2}+G\left(x_{1}\right)=u, \\
& M\left(x_{1}\right) \dot{x}_{2}=-C\left(x_{1}, x_{2}\right) x_{2}-G\left(x_{1}\right)+u .
\end{aligned}
$$

Consequently, a change of variables is used as follows:

$$
\begin{gathered}
M\left(x_{1}\right) \dot{x}_{2}=-\widehat{C}\left(x_{1}, x_{2}\right) x_{2}-N\left(x_{1}, x_{2}\right)+u, \\
x^{T}\left[\dot{M}\left(x_{1}\right)-2 \widehat{C}\left(x_{1}, x_{2}\right)\right] x=0,
\end{gathered}
$$

where the elements are given in (12). Note that the elements of $\widehat{C}\left(x_{1}, x_{2}\right)$ and $N\left(x_{1}, x_{2}\right)$ are selected such that the property (14) is satisfied.

In the following section, a stable controller for the pendulum systems will be designed.

\section{Proportional Derivative Control with Inverse Dead-Zone}

The regulation case is considered in this study; that is, the desired velocity is $x_{2}^{d}=0$. The proportional derivative control with inverse dead-zone $v$ is as follows:

$$
v=\mathrm{DZ}^{-1}\left(u_{\mathrm{pd}}\right)= \begin{cases}\frac{1}{m_{r}} u_{\mathrm{pd}}+b_{r} & u_{\mathrm{pd}}>0 \\ 0 & u_{\mathrm{pd}}=0 \\ \frac{1}{m_{l}} u_{\mathrm{pd}}+b_{l} & u_{\mathrm{pd}}<0\end{cases}
$$

where the parameters $m_{r}, m_{l}, b_{r}$, and $b_{l}$ are defined as in (2), and the auxiliary proportional derivative control is

$$
u_{\mathrm{pd}}=-K_{p} \tilde{x}_{1}-K_{d} \tilde{x}_{2}+\widehat{N}-K \operatorname{sign}\left(\tilde{x}_{2}\right),
$$

where $\tilde{x}_{1}=x_{1}-x_{1}^{d}$ is the tracking error, $x_{1}$ and $\tilde{x}_{2}=x_{2}$ are defined in (3), $x_{1}^{d}=\left[\begin{array}{ll}x_{11}^{d} & x_{12}^{d}\end{array}\right] \in \mathfrak{R}^{n \times 1}$ is the desired position, $K_{p}, K_{d}$ are positive definite, $\widehat{N} \in \Re^{n \times 1}$ is an approximation of $N\left(x_{1}, x_{2}\right)$, and $N\left(x_{1}, x_{2}\right) \in \Re^{n \times 1}$ are the nonlinear terms of (12). Figure 2 shows the inverse dead-zone [17, 22] and Figure 3 shows the proposed controller denoted as PDDZ. It is considered that the approximation error $\widetilde{N}=\widehat{N}-N\left(x_{1}, x_{2}\right)$ is bounded as

$$
|\widetilde{N}| \leq \bar{N}
$$

Now the convergence of the closed-loop system is discussed.

Theorem 3. The error of the closed-loop system with the proportional derivative control (17) and (18) for the pendulum systems with dead-zone inputs (12) and (5) is asymptotically stable, and the error of the velocity parameter $\tilde{x}_{2}$ will converge to

$$
\limsup _{T \rightarrow \infty}\left\|\tilde{x}_{2}\right\|^{2}=0,
$$

where $T$ is the final time, $\tilde{x}_{2}=x_{2}, \bar{N} \leq K, K_{p}>0$, and $K_{d}>0$.

Proof. The proposed Lyapunov function is

$$
V_{1}=\frac{1}{2} \tilde{x}_{2}^{T} M\left(x_{1}\right) \tilde{x}_{2}+\frac{1}{2} \widetilde{x}_{1}^{T} K_{p} \tilde{x}_{1} .
$$

Substituting (17) and (18) into (12) and (5) the closed-loop system is as follows:

$$
\begin{aligned}
M\left(x_{1}\right) \dot{x}_{2}= & -K_{p} \widetilde{x}_{1}-K_{d} x_{2}+\widetilde{N} \\
& -K \operatorname{sign}\left(x_{2}\right)-\widehat{C}\left(x_{1}, x_{2}\right) x_{2} .
\end{aligned}
$$

Using the fact $\tilde{x}_{2}=x_{2}$, the derivative of (21) is

$$
\dot{V}_{1}=\tilde{x}_{2}^{T} M\left(x_{1}\right) \dot{x}_{2}+\frac{1}{2} \tilde{x}_{2}^{T} \dot{M}\left(x_{1}\right) \tilde{x}_{2}+\tilde{x}_{2}^{T} K_{p} \tilde{x}_{1},
$$




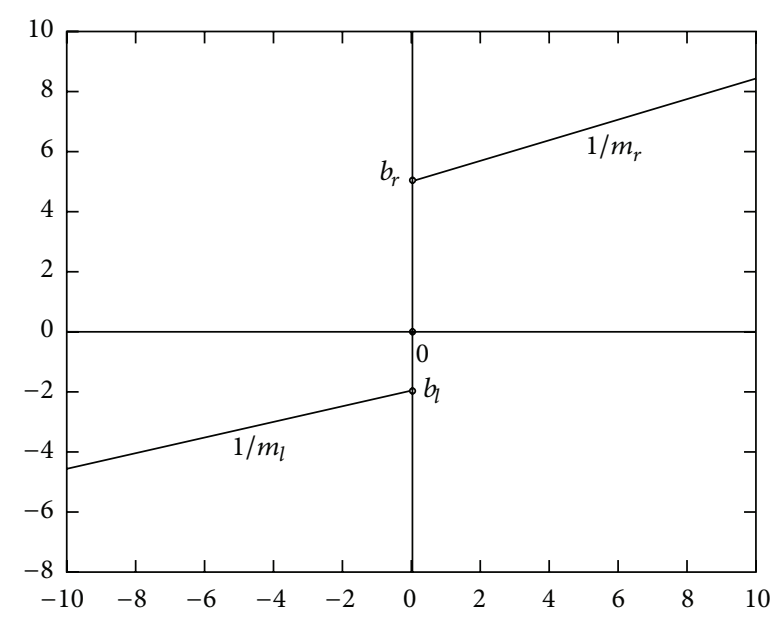

Figure 2: The inverse dead-zone.

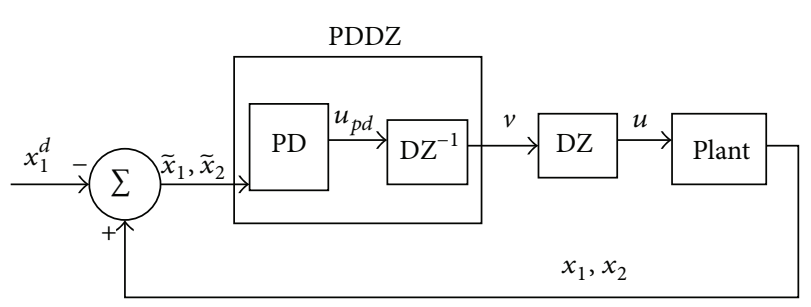

FIGURE 3: The proposed controller.

where $\dot{\tilde{x}}_{1}=\dot{x}_{1}-\dot{x}_{1}^{d}=x_{2}-x_{2}^{d}=x_{2}=\widetilde{x}_{2}$ and $\dot{\tilde{x}}_{2}=\dot{x}_{2}$. Substituting (22) into (23) gives

$$
\begin{aligned}
\dot{V}_{1}= & -\widetilde{x}_{2}^{T} K_{d} \widetilde{x}_{2}+\widetilde{x}_{2}^{T}\left[\widetilde{N}-K \operatorname{sign}\left(x_{2}\right)\right] \\
& +\frac{1}{2} \widetilde{x}_{2}^{T}\left[\dot{M}\left(x_{1}\right)-2 \widehat{C}\left(x_{1}, x_{2}\right)\right] \widetilde{x}_{2}^{T} .
\end{aligned}
$$

Using (14), (17), and $\left|\widetilde{x}_{2}\right|^{T}=\widetilde{x}_{2}^{T} \operatorname{sign}\left(x_{2}\right)$, it gives

$$
\begin{gathered}
\dot{V}_{1} \leq-\widetilde{x}_{2}^{T} K_{d} \widetilde{x}_{2}+\left|\widetilde{x}_{2}\right|^{T} \bar{N}-\left|\widetilde{x}_{2}\right|^{T} K, \\
\dot{V}_{1} \leq-\widetilde{x}_{2}^{T} K_{d} \tilde{x}_{2},
\end{gathered}
$$

where $\bar{N} \leq K$. Thus, the error is asymptotically stable [26]. Integrating (25) from 0 to $T$ yields

$$
\begin{gathered}
\int_{0}^{T} \tilde{x}_{2}^{T} K_{d} \tilde{x}_{2} d t \leq V_{1,0}-V_{1, T} \leq V_{1,0}, \\
\frac{1}{T} \int_{0}^{T} \tilde{x}_{2}^{T} K_{d} \tilde{x}_{2} d t \leq \frac{1}{T} V_{1,0}, \\
\limsup _{T \rightarrow \infty}\left(\frac{1}{T} \int_{0}^{T} \tilde{x}_{2}^{T} K_{d} \tilde{x}_{2} d t\right) \leq V_{1,0}\left[\limsup _{T \rightarrow \infty}\left(\frac{1}{T}\right)\right]=0 .
\end{gathered}
$$

If $T \rightarrow \infty$, then $\left\|\widetilde{x}_{2}\right\|^{2}=0$; (20) is established.

Remark 4. The proposed controller is used for the regulation case; that is, the desired velocity is $x_{2}^{d}=0$. The general case when $x_{2}^{d} \neq 0$ is not considered in this research.

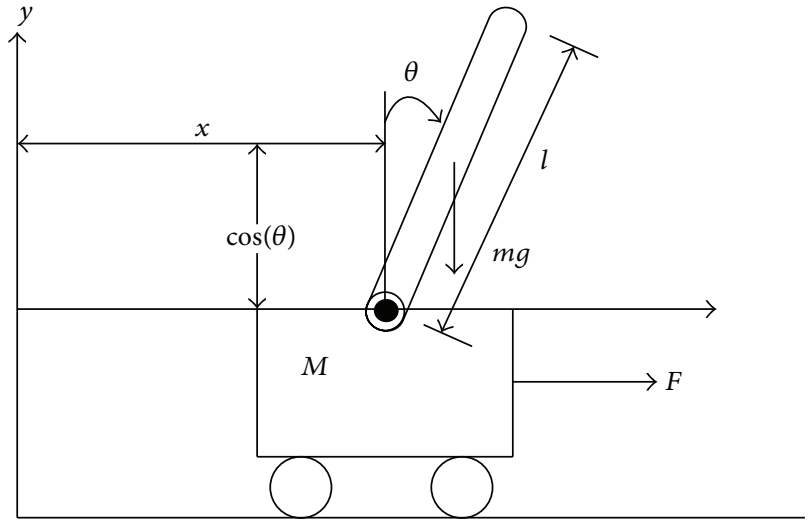

FIGURE 4: Inverted-car pendulum.

\section{Simulations}

In this section, the proportional derivative control with inverse dead-zone denoted as PDDZ will be compared with the proportional derivative control with gravity compensation of [23] denoted by PD for the control of two pendulum systems with dead-zone inputs. In this paper, the root mean square error (RMSE) $[1,26,27]$ is used for the comparison results and it is given as

$$
\operatorname{RMSE}=\left(\frac{1}{T} \int_{0}^{T} \tilde{x}^{2} d t\right)^{1 / 2},
$$

where $\tilde{x}^{2}=\tilde{x}_{12}^{2}$ or $\tilde{x}^{2}=u_{1}^{2}$.

5.1. Example 1. Consider the inverted-car pendulum [2] of Figure 4.

Inverted-car pendulum is written as (1) and it is detailed as follows:

$$
\begin{gathered}
M\left(x_{1}\right)=\left[\begin{array}{ll}
m_{11} & m_{12} \\
m_{21} & m_{22}
\end{array}\right], \\
C\left(x_{1}, x_{2}\right)=\left[\begin{array}{ll}
c_{11} & c_{12} \\
c_{21} & c_{22}
\end{array}\right], \\
G\left(x_{1}\right)=\left[\begin{array}{l}
g_{1} \\
g_{2}
\end{array}\right],
\end{gathered}
$$

where $m_{11}=M+m, m_{12}=m_{21}=m l \cos \left(x_{12}\right), m_{33}=$ $I+m l^{2}, c_{12}=-m l \sin \left(x_{12}\right) x_{22}$, the other parameters of $C\left(x_{1}\right.$, $x_{2}$ ) are zero, $g_{2}=-m g l \sin \left(x_{12}\right)$, and the other parameter of $G\left(x_{1}\right)$ is zero. $\sin (\cdot)$ is the sine function, $\cos (\cdot)$ is the cosine function, $I=0.5 \mathrm{kgm}^{2}$ is the pendulum inertia, $M=$ $0.136729 \mathrm{~kg}$ is the mass of the car, $m=0.040691 \mathrm{~kg}$ is the pendulum mass, $l=0.15 \mathrm{~m}$ is the pendulum length, $\theta_{12}$ is the angle with respect of the $y$ axis, $u=F$ is the motion force of the car, $x_{11}=x$ is the motion distance of the car, and $g=9.81 \mathrm{~m} / \mathrm{s}^{2}$ is the constant acceleration due to gravity. It can be proven that Property 2 of (7) is not satisfied. 


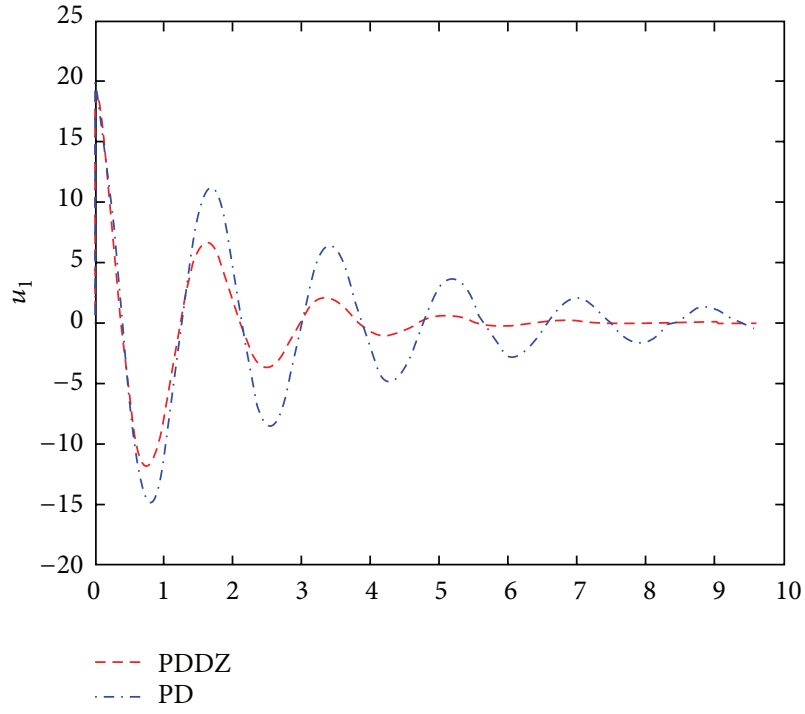

FIGURE 5: Input for Example 1.

Inverted-car pendulum is written as (12), and it is detailed as follows:

$$
\begin{gathered}
M\left(x_{1}\right)=\left[\begin{array}{ll}
m_{11} & m_{12} \\
m_{21} & m_{22}
\end{array}\right], \\
\widehat{C}\left(x_{1}, x_{2}\right)=\left[\begin{array}{ll}
\widehat{c}_{11} & \widehat{c}_{12} \\
\widehat{c}_{21} & \widehat{c}_{22}
\end{array}\right], \\
N\left(x_{1}, x_{2}\right)=\left[\begin{array}{l}
n_{1} \\
n_{2}
\end{array}\right],
\end{gathered}
$$

where $m_{11}=M+m, m_{12}=m_{21}=m l \cos \left(x_{12}\right), m_{33}=$ $I+m l^{2}, \widehat{c}_{12}=\widehat{c}_{21}=-(1 / 2) m l \sin \left(x_{12}\right) x_{22}$ and the other parameters of $\widehat{C}\left(x_{1}, x_{2}\right)$ are zero, $n_{1}=-(1 / 2) m l \sin \left(x_{12}\right) x_{22}$, $n_{2}=-m g l \sin \left(x_{12}\right)+(1 / 2) m l \sin \left(x_{12}\right) x_{22}$; therefore, it can be proven that the property of the lemma of (14) is satisfied.

PDDZ is given by (17) and (18) as $u_{\mathrm{pd}}=-K_{p} \widetilde{x}_{12}-K_{d} \widetilde{x}_{22}+$ $\widehat{n}_{1}-K \operatorname{sign}\left(\widetilde{x}_{22}\right)$ with parameters $K_{p}=200, K_{d}=20, \widehat{N}=\widehat{n}_{1}$, and $\widehat{n}_{1}=0.01$, with $K=0.01$. Conditions given in (20) $\bar{N} \leq K, K_{p}>0$, and $K_{d}>0$ are satisfied; consequently, the error of the closed-loop dynamics of the PDDZ applied for pendulum systems is guaranteed to be asymptotically stable.

$\mathrm{PD}$ is given by [23] as $u_{\mathrm{pd}}=-K_{p} \widetilde{x}_{12}-K_{d} \widetilde{x}_{22}+g_{2}$ with parameters $K_{p}=400, K_{d}=20$, and $G\left(x_{1}\right)=g_{1}$.

Comparison results for the control functions are shown in Figure 5, position states are shown in Figure 6, and comparison results for the controller errors are shown in Figure 7. Comparison of the square norm of the velocity errors $\left\|\tilde{x}_{2}\right\|^{2}$ of (20) for the controllers is presented in Figure 8. From the theorem of (20), $\left\|\tilde{x}_{2}\right\|^{2}$ will converge to zero for the PDDZ. Table 1 shows the RMSE results using (27).

The most important variable to control is the pendulum angle $x_{12}=\theta$, and this variable may reach zero even if it starts with other value as in this example. Note that the PD technique requires the bigger gains than the PDDZ method to obtain satisfactory results. From Figures 5, 6, and 7, it can

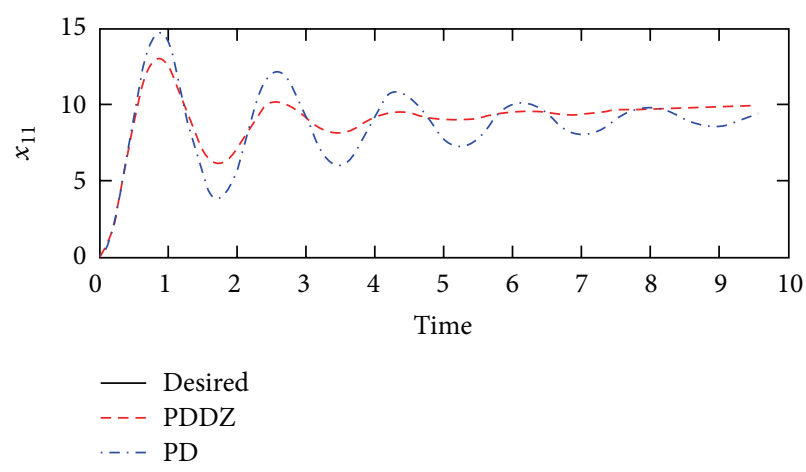

(a)

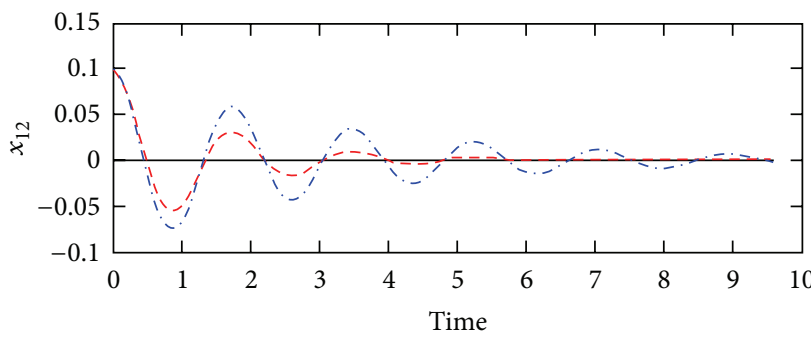

_ Desired
--- PDDZ
$-. \cdot-$ PD

(b)

Figure 6: Position states for Example 1.

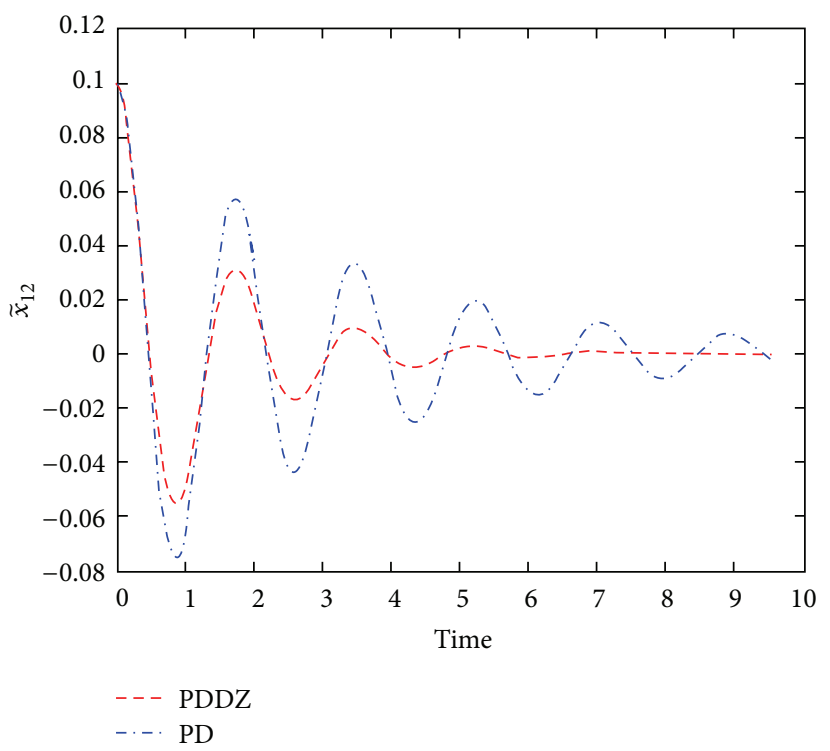

FIgURE 7: Position state error for Example 1.

be seen that the PDDZ improves the PD because the signal of the plant for the first follows better the desired signal than the second and in the first the inputs are smaller than in the second. From Figure 8, it is shown that the PDDZ improves the PD because the velocity error $\left\|\widetilde{x}_{2}\right\|^{2}$ presented by the first is smaller than that presented by the second. From Table 1, it 


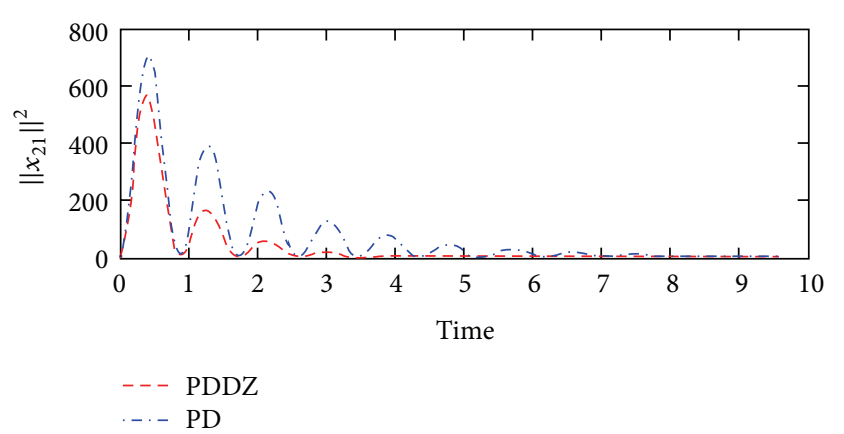

(a)

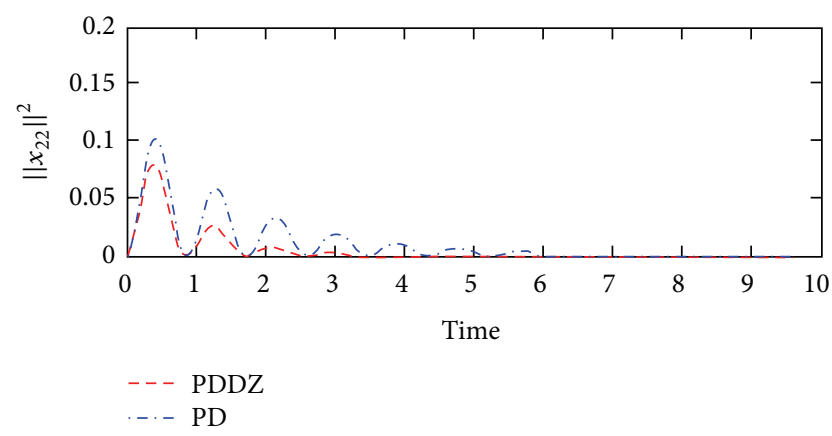

(b)

FIGURE 8: Velocity errors for Example 1.

TABLE 1: Error results for Example 1.

\begin{tabular}{lcc}
\hline Methods & RMSE for $\tilde{x}$ & RMSE for $u$ \\
\hline PD & $4.2487 \times 10^{-4}$ & 15.5044 \\
PDDZ & $2.1995 \times 10^{-4}$ & 7.7534 \\
\hline
\end{tabular}

can be shown that the PDDZ achieves better accuracy when compared with the PD because the RMSE is smaller for the first than for the second.

5.2. Example 2. Consider the Furuta pendulum $[3,27]$ of the Figure 9. follows:

Furuta pendulum is written as (1) and it is detailed as

$$
\begin{gathered}
M\left(x_{1}\right)=\left[\begin{array}{ll}
m_{11} & m_{12} \\
m_{21} & m_{22}
\end{array}\right], \\
C\left(x_{1}, x_{2}\right)=\left[\begin{array}{ll}
l_{11} & c_{12} \\
c_{21} & c_{22}
\end{array}\right], \\
G\left(x_{1}\right)=\left[\begin{array}{l}
g_{1} \\
g_{2}
\end{array}\right],
\end{gathered}
$$

where $m_{11}=I_{0}+m_{1}\left(L_{0}^{2}+l_{1}^{2} \sin ^{2}\left(x_{12}\right)\right), m_{12}=m_{21}=m_{1} l_{1} L_{0}$ $\cos \left(x_{12}\right), m_{33}=J_{1}+m_{1} l_{1}^{2}, c_{11}=m_{1} l_{1}^{2} \sin \left(2 x_{12}\right) x_{22}, c_{12}=$ $-m_{1} l_{1} L_{0} \sin \left(x_{12}\right) x_{22}, c_{21}=-m_{1} l_{1}^{2} \sin \left(x_{12}\right) \cos \left(x_{12}\right) x_{21}, g_{2}=$ $-m_{1} g l_{1} \sin \left(x_{12}\right)$, and the other parameter of $G\left(x_{1}\right)$ is zero. $\sin (\cdot)$ is the sine function, $\cos (\cdot)$ is the cosine function, $I_{0}=$ $0.5 \mathrm{Kgm}^{2}$ is the arm inertia, $J_{1}=0.5 \mathrm{kgm}^{2}$ is the pendulum inertia, $m_{2}=0.34 \mathrm{~kg}$ is the arm mass, $m_{1}=0.24 \mathrm{~kg}$ is the

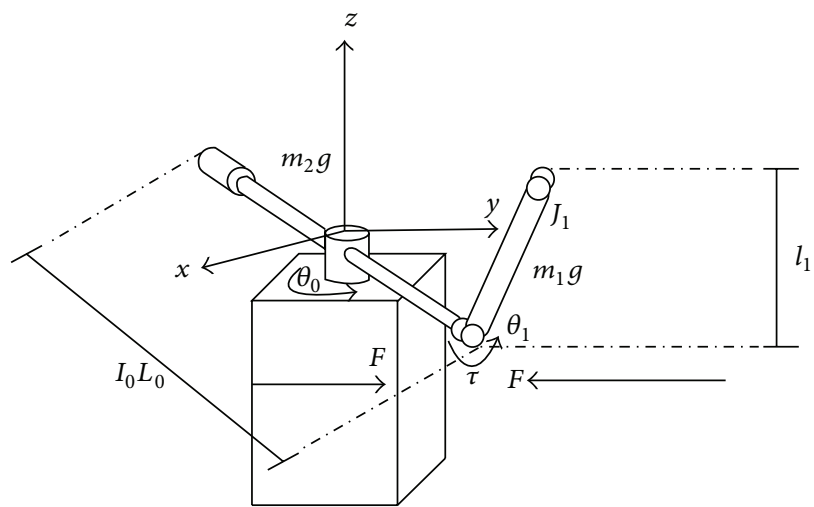

FIgURE 9: Furuta pendulum.

pendulum mass, $L_{0}=0.293 \mathrm{~m}$ is the arm length, $l_{1}=0.28 \mathrm{~m}$ is the pendulum length, $x_{11}=\theta_{0}$ is the arm angle, $x_{12}=$ $\theta_{1}$ is the pendulum angle, $u=F$ is the motion torque of the arm, and $g=9.81 \mathrm{~m} / \mathrm{s}^{2}$ is the constant acceleration due to gravity. It can be proven that Property 2 of (7) is not satisfied.

Furuta pendulum is written as (12), and it is detailed as follows:

$$
\begin{gathered}
M\left(x_{1}\right)=\left[\begin{array}{ll}
m_{11} & m_{12} \\
m_{21} & m_{22}
\end{array}\right], \\
\widehat{C}\left(x_{1}, x_{2}\right)=\left[\begin{array}{ll}
\widehat{c}_{11} & \widehat{c}_{12} \\
\widehat{c}_{21} & \widehat{c}_{22}
\end{array}\right], \\
N\left(x_{1}, x_{2}\right)=\left[\begin{array}{l}
n_{1} \\
n_{2}
\end{array}\right],
\end{gathered}
$$

where $m_{11}=M+m, m_{12}=m_{21}=m l \cos \left(x_{12}\right), m_{33}=I+m l^{2}$, $\widehat{c}_{11}=m_{1} l_{1}^{2} \sin \left(x_{12}\right) \cos \left(x_{12}\right) x_{22}, \widehat{c}_{12}=-(1 / 2) m_{1} l_{1} L_{0} \sin \left(x_{12}\right)$ $x_{22}, \widehat{c}_{21}=-(1 / 2) m_{1} l_{1}^{2} \sin \left(x_{12}\right) \cos \left(x_{12}\right) x_{22}$, and the other parameter of $\widehat{C}\left(x_{1}, x_{2}\right)$ is zero, $n_{1}=m_{1} l_{1}^{2} \sin \left(x_{12}\right) \cos \left(x_{12}\right)$ $x_{22}-(1 / 2) m_{1} l_{1} L_{0} \sin \left(x_{12}\right) x_{22}, n_{2}=-m_{1} g l_{1} \sin \left(x_{12}\right)-(1 / 2)$ $m_{1} l_{1}^{2} \sin \left(x_{12}\right) \cos \left(x_{12}\right)\left(x_{21}-x_{22}\right)$; therefore, it can be proven that the property of the lemma of (14) is satisfied.

PDDZ is given by (17) and (18) as $u_{\mathrm{pd}}=-K_{p} \widetilde{x}_{12}-K_{d} \tilde{x}_{22}+$ $\widehat{n}_{1}-K \operatorname{sign}\left(\tilde{x}_{22}\right)$ with parameters $K_{p}=200, K_{d}=20, \widehat{N}=\widehat{n}_{1}$, $\widehat{n}_{1}=0.01$, with $K=0.01$. Conditions given in (20) $\bar{N} \leq K$, $K_{p}>0$, and $K_{d}>0$ are satisfied; consequently, the error of the closed-loop dynamics of the PDDZ applied for pendulum systems is guaranteed to be asymptotically stable.

$\mathrm{PD}$ is given by [23] as $u_{\mathrm{pd}}=-K_{p} \tilde{x}_{12}-K_{d} \tilde{x}_{22}+g_{2}$ with parameters $K_{p}=400, K_{d}=20$, and $G\left(x_{1}\right)=g_{2}$.

Comparison results for the control functions are shown in Figure 10, position states are shown in Figure 11, and comparison results for the controller errors are shown in Figure 12. Comparison of the square norm of the velocity errors $\left\|\tilde{x}_{2}\right\|^{2}$ of (20) for the controllers is presented in Figure 13. From the theorem of (20), $\left\|\tilde{x}_{2}\right\|^{2}$ will converge to zero for the PDDZ. Table 2 shows the RMSE results using (27).

The most important variable to control is the pendulum angle $x_{12}=\theta_{1}$, and this variable may reach zero even if it 


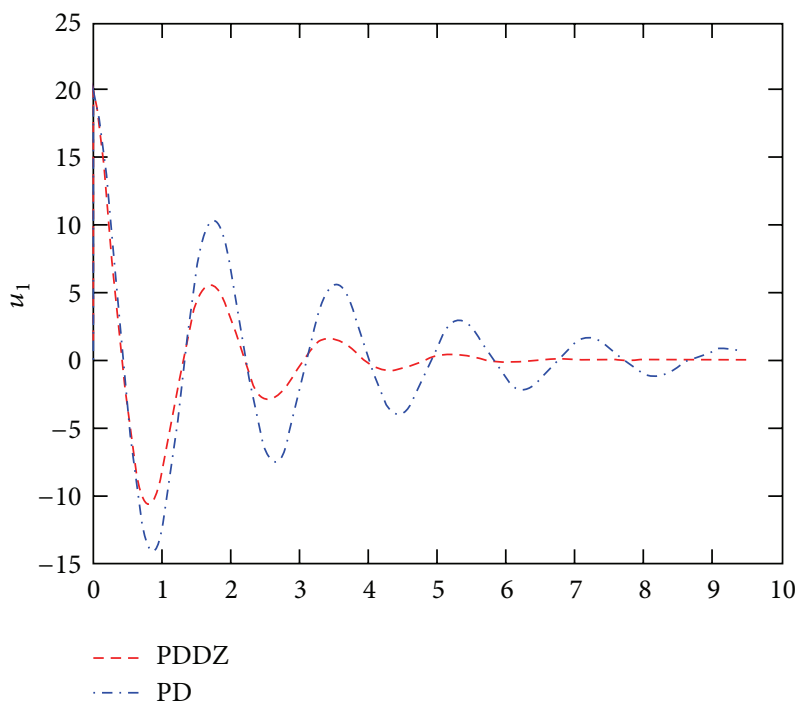

FIgURE 10: Input for Example 2.

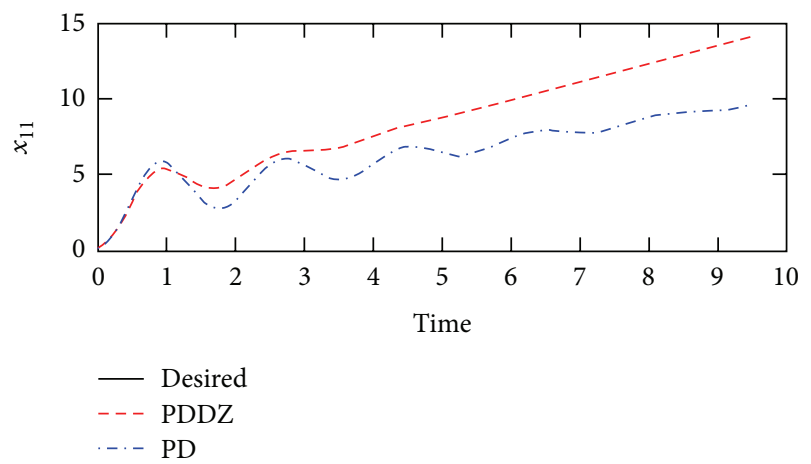

(a)

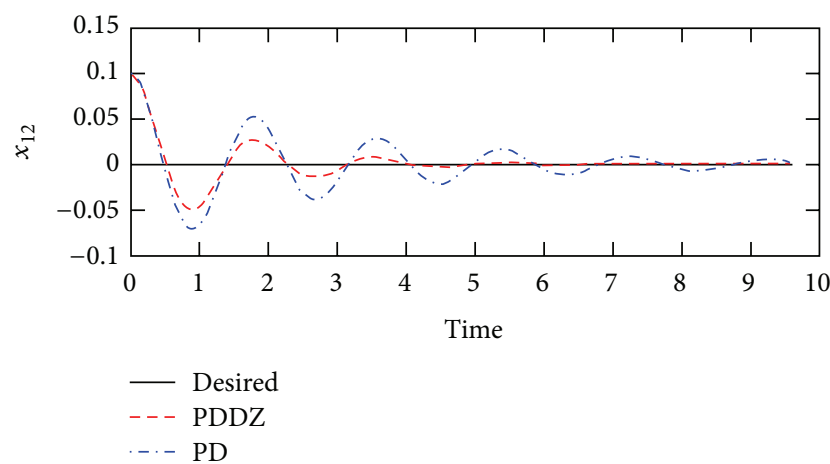

(b)

Figure 11: Position states for Example 2.

starts with other value as in this example. Note that the PD technique requires the bigger gains than the PDDZ method to obtain satisfactory results. From Figures 10, 11, and 12, it can be seen that the PDDZ improves the PD because the signal of the plant for the first follows better the desired signal than the second and in the first the inputs are smaller than in the

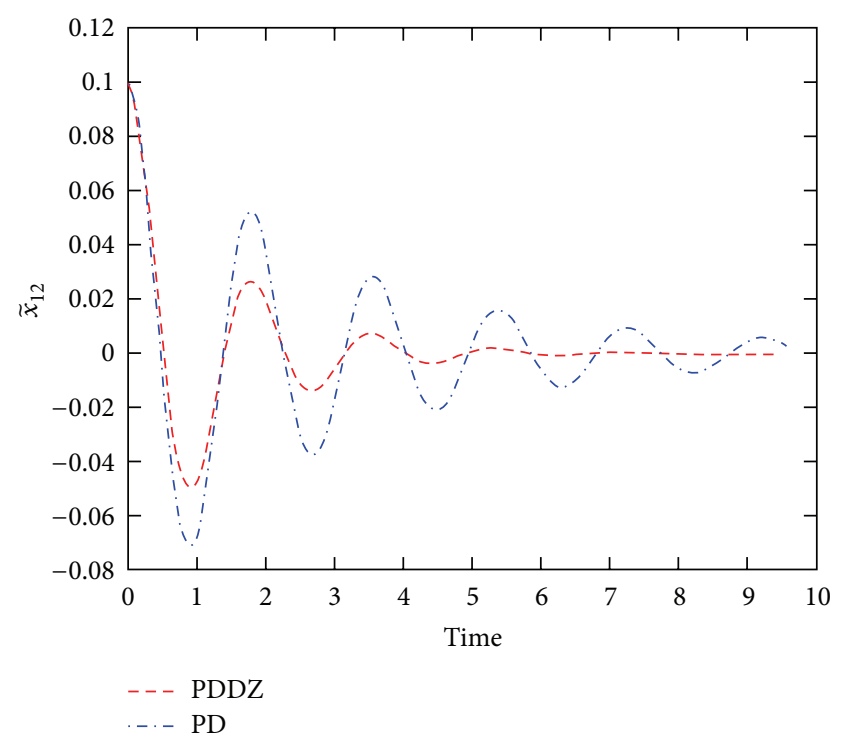

FIGURE 12: Position state error for Example 2.

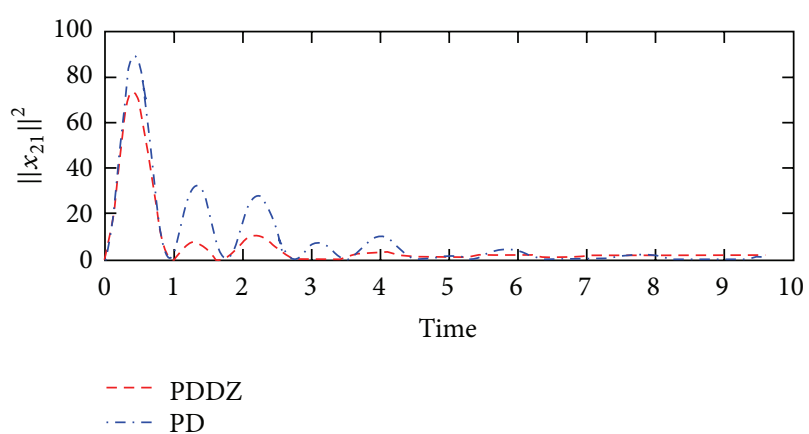

(a)

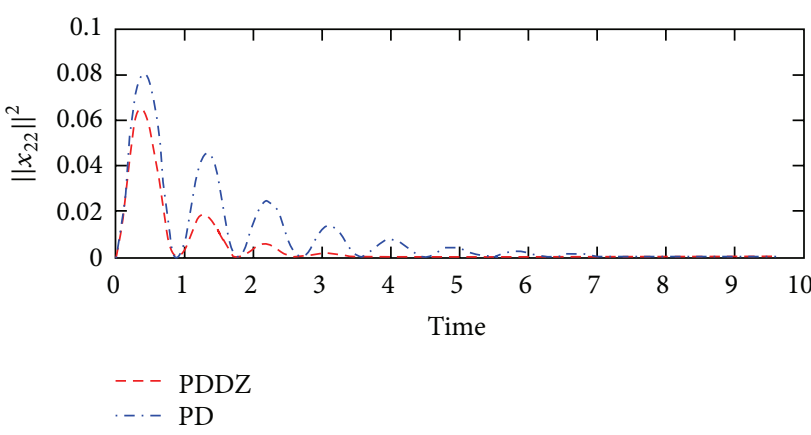

(b)

Figure 13: Velocity errors for Example 2.

second. From Figure 13, it is shown that the PDDZ improves the PD because the velocity error $\left\|\widetilde{x}_{2}\right\|^{2}$ presented by the first is smaller than that presented by the second. From Table 2, it can be shown that the PDDZ achieves better accuracy when compared with the PD because the RMSE is smaller for the first than for the second. 
TABLE 2: Error results for Example 2.

\begin{tabular}{lcc}
\hline Methods & RMSE for $\tilde{x}$ & RMSE for $u$ \\
\hline PD & $3.7760 \times 10^{-4}$ & 13.6043 \\
PDDZ & $2.0158 \times 10^{-4}$ & 6.8328 \\
\hline
\end{tabular}

\section{Conclusion}

In this research, a proportional derivative control with inverse dead-zone for pendulum systems with dead-zone inputs is presented. The simulations showed that the proposed technique achieves better performance when compared with the proportional derivative control with gravity compensation for the regulation of two pendulum systems, and the results illustrate the viability, efficiency, and the potential of the approach especially important in pendulum systems. As a future research, the proposed study will be improved considering that some parameters of the controller are unknown [28-32], or it will consider the communication delays and packet dropout.

\section{Conflict of Interests}

The authors declare no conflict of interests about all the aspects related to this paper.

\section{Acknowledgments}

The authors are grateful to the editors and the reviewers for their valuable comments and insightful suggestions, which helped to improve this research significantly. The authors thank the Secretaría de Investigación y Posgrado, the Comisión de Operación y Fomento de Actividades Académicas del IPN, and Consejo Nacional de Ciencia y Tecnología for their help in this research.

\section{References}

[1] J. J. Rubio and J. H. Pérez-Cruz, "Evolving intelligent system for the modelling of nonlinear systems with dead-zone input," Applied Soft Computing. In press.

[2] C. Aguilar-Ibañez, J. C. Martínez-García, A. Soria-López, and J. D. J. Rubio, "On the stabilization of the inverted-cart pendulum using the saturation function approach," Mathematical Problems in Engineering, vol. 2011, Article ID 856015, 14 pages, 2011.

[3] C. Aguilar-Ibañez, M. S. Suárez-Castañón, and O. O. GutiérresFrias, "The direct Lyapunov method for the stabilisation of the furuta pendulum," International Journal of Control, vol. 83, no. 11, pp. 2285-2293, 2010.

[4] D. Ding, Z. Wang, J. Hu, and H. Shu, "Dissipative control for state-saturated discrete time-varying systems with randomly occurring nonlinearities and missing measurements," International Journal of Control, vol. 86, no. 4, pp. 674-688, 2013.

[5] H. Dong, Z. Wang, J. Lam, and H. Gao, "Distributed filtering in sensor networks with randomly occurring saturations and successive packet dropouts," International Journal of Robust and Nonlinear Control, 2013.
[6] J. Hu, Z. Wang, B. Shen, and H. Gao, "Quantised recursive filtering for a class of nonlinear systems with multiplicative noises and missing measurements," International Journal of Control, vol. 86, no. 4, pp. 650-663, 2013.

[7] J. Hu, Z. Wang, B. Shen, and H. Gao, "Gain-constrained recursive filtering with stochastic nonlinearties and probabilistic sensor delays," IEEE Transactions on Signal Processing, vol. 61, no. 5, pp. 1230-1238, 2013.

[8] M. Jimenez-Lizarraga, M. Basin, and P. Rodriguez-Ramirez, "Robust mini-max regulator for uncertain non-linear polynomial systems," IET Control Theory \& Applications, vol. 6, no. 7, pp. 963-970, 2012.

[9] A. F. de Loza, M. Jimenez-Lizarraga, and L. Fridman, "Robust output nash strategies based on sliding mode observation in a two-player differential game," Journal of the Franklin Institute, vol. 349, no. 4, pp. 1416-1429, 2012.

[10] Q. Lu, L. Zhang, H. R. Karimi, and Y. Shi, " $H_{\infty}$ control for asynchronously switched linear parameter-varying systems with mode-dependent average dwell time," IET Control Theory \& Applications, vol. 7, no. 5, pp. 677-683, 2013.

[11] L. F. Ma, Z. D. Wang, and Z. Guo, "Robust $\mathrm{H}_{2}$ sliding mode control for non-linear discrete-time stochastic systems," IET Control Theory and Applications, vol. 3, no. 11, pp. 1537-1546, 2009.

[12] Y. Niu, D. W. C. Ho, and Z. Wang, "Improved sliding mode control for discrete-time systems via reaching law," IET Control Theory \& Applications, vol. 4, no. 11, pp. 2245-2251, 2010.

[13] L. A. Soriano, W. Yu, and J. J. Rubio, "Modeling and control of wind turbine," Mathematical Problems in Engineering, vol. 2013, Article ID 982597, 13 pages, 2013.

[14] J. J. Rubio, G. Ordaz, M. Jimenez-Lizarraga, and R. I. Cabrera, "General solution to the Navier-Stokes equation to describe the dynamics of a homogeneous viscous fluid in an open pipe," Revista Mexicana de Fisica, vol. 59, no. 3, pp. 217-223, 2013.

[15] Y. Wang, H. R. Karimi, and Z. Xiang, "Delay-dependent $H_{\infty}$ control for networked control systems with large delays," Mathematical Problems in Engineering, vol. 2013, Article ID 643174, 10 pages, 2013.

[16] Y. Wang, H. R. Karimi, and Z. Xiang, " $H_{\infty}$ control for networked control systems with time delays and packet dropouts," Mathematical Problems in Engineering, vol. 2013, Article ID 635941, 10 pages, 2013.

[17] S. Ibrir, W. F. Xie, and C.-Y. Su, "Adaptive tracking of nonlinear systems with non-symmetric dead-zone input," Automatica, vol. 43, no. 3, pp. 522-530, 2007.

[18] S. Abrir, "Invarian-manifold approach to the stabilization of feedforward nonlinear systems having uncertain dead-zone inputs," in Proceedings of the International Sysmposium of Mechatronics and Its Applications, pp. 1-5, 2012.

[19] J. H. Pérez-Cruz, E. Ruiz-Velázquez, J. J. Rubio, and C. A. de Alva Padilla, "Robust adaptive neurocontrol of SISO nonlinear systems preceded by unknow deadzone," Mathematical Problems in Engineering, vol. 2012, Article ID 342739, 23 pages, 2012.

[20] J. H. Pérez-Cruz, J. J. Rubio, E. Ruiz-Velázquez, and G. SolsPerales, "Tracking control based on recurrent neural networks for nonlinear systems with multiple inputs and unknown deadzone," Abstract and Applied Analysis, vol. 2012, Article ID 471281, 18 pages, 2012.

[21] Z. Wang, Y. Zhang, and H. Fang, "Neural adaptive control for a class of nonlinear systems with unknown deadzone," Neural Computing and Applications, vol. 17, no. 4, pp. 339-345, 2008. 
[22] J. Zhou and X. Z. Shen, "Robust adaptive control of nonlinear uncertain plants with unknown dead-zone," IET Control Theory and Applications, vol. 1, no. 1, pp. 25-32, 2007.

[23] F. L. Lewis, D. M. Dawson, and C. T. Abdallah, Control of Robot Manipulators, Theory and Practice, CRC Press, New York, NY, USA, 2004.

[24] J. E. Slotine and W. Li, Applied Nonlinear Control, Macmillan, Englewood Clifs, NJ, USA, 1991.

[25] M. W. Spong and M. Vidyasagar, Robot Dynamics and Control, John Wiley \& Sons, 1989.

[26] J. J. Rubio, G. Gutierrez, J. Pacheco, and J. H. Pérez-Cruz, "Comparison of three proposed controls to accelerate the growth of the crop," International Journal of Innovative Computing, Information and Control, vol. 7, no. 7, pp. 4097-4114, 2011.

[27] J. J. Rubio, M. Figueroa, J. H. Pérez-Cruz, and J. Rumbo, "Control to stabilize and mitigate disturbances in a rotary inverted pendulum," Revista Mexicana de Fisica E, vol. 58, no. 2, pp. 107-112, 2012.

[28] J. A. Iglesias, P. Angelov, A. Ledezma, and A. Sanchis, "Creating evolving user behavior profiles automatically," IEEE Transactions on Knowledge and Data Engineering, vol. 24, no. 5, pp. 854867, 2011.

[29] D. Leite, R. Ballini, P. Costa, and F. Gomide, "Evolving fuzzy granular modeling from nonstationary fuzzy data streams," Evolving Systems, vol. 3, no. 2, pp. 65-79, 2012.

[30] E. Lughofer, "Single pass active learning with conflict and ignorance," Evolving Systems, vol. 3, pp. 251-271, 2012.

[31] E. Lughofer, "A dynamic split-and-merge approach for evolving cluster models," Evolving Systems, vol. 3, pp. 135-151, 2012.

[32] L. Maciel, A. Lemos, F. Gomide, and R. Ballini, "Evolving fuzzy systems for pricing fixed income options," Evolving Systems, vol. 3, no. 1, pp. 5-18, 2012. 


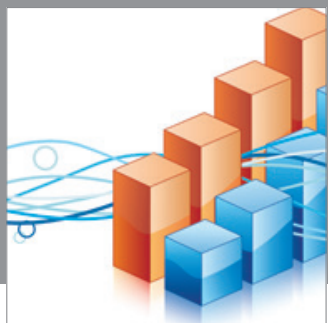

Advances in

Operations Research

mansans

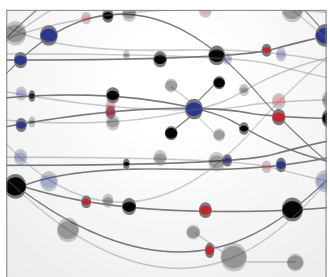

The Scientific World Journal
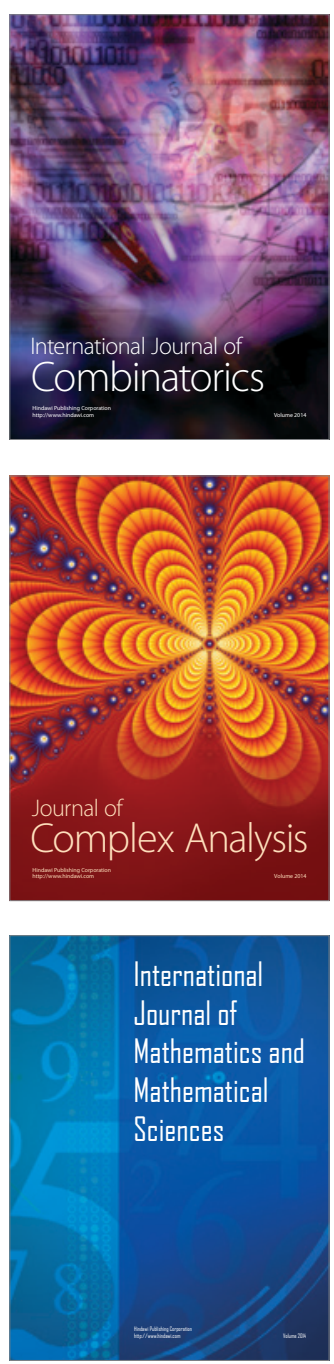
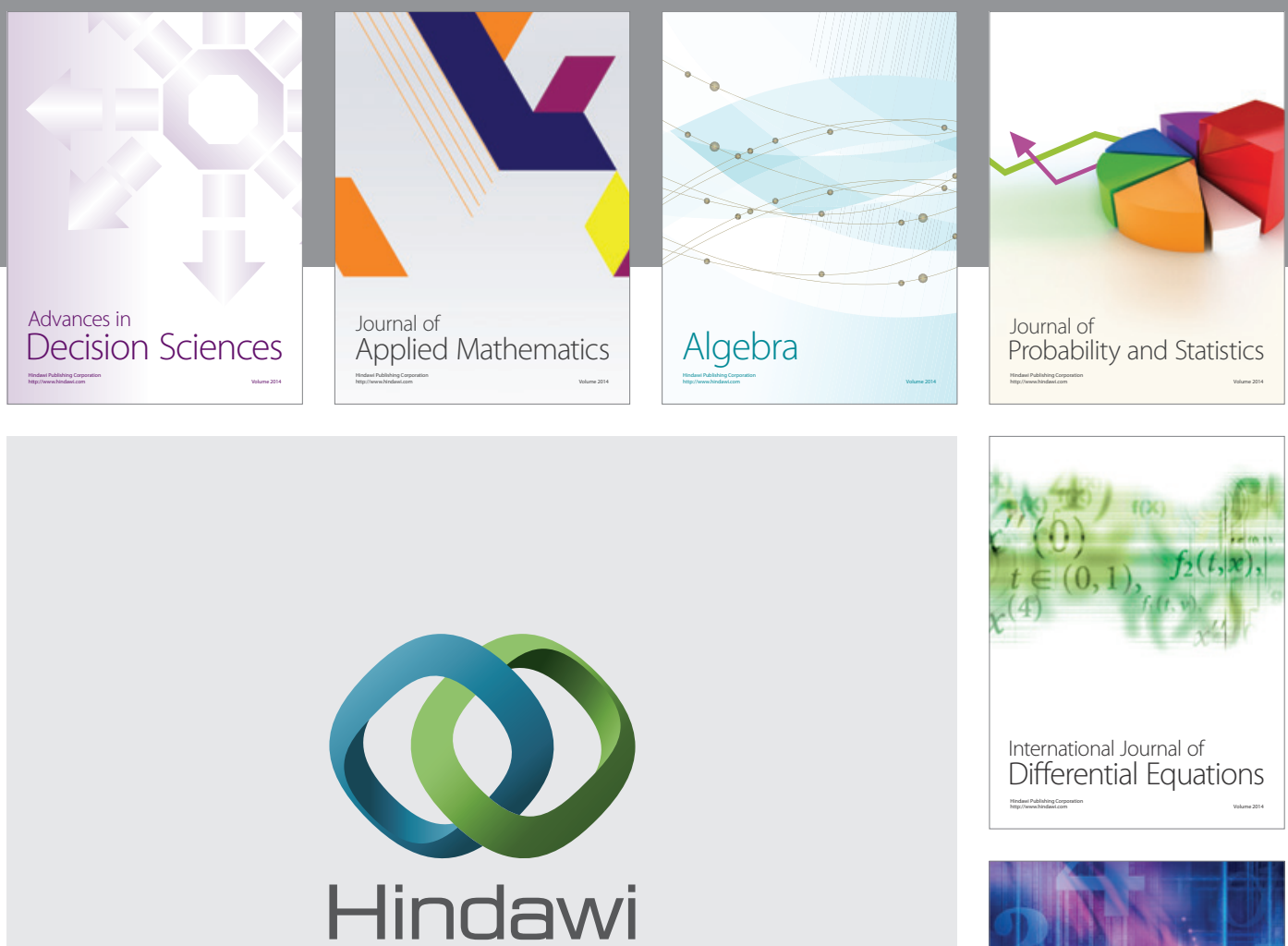

Submit your manuscripts at http://www.hindawi.com
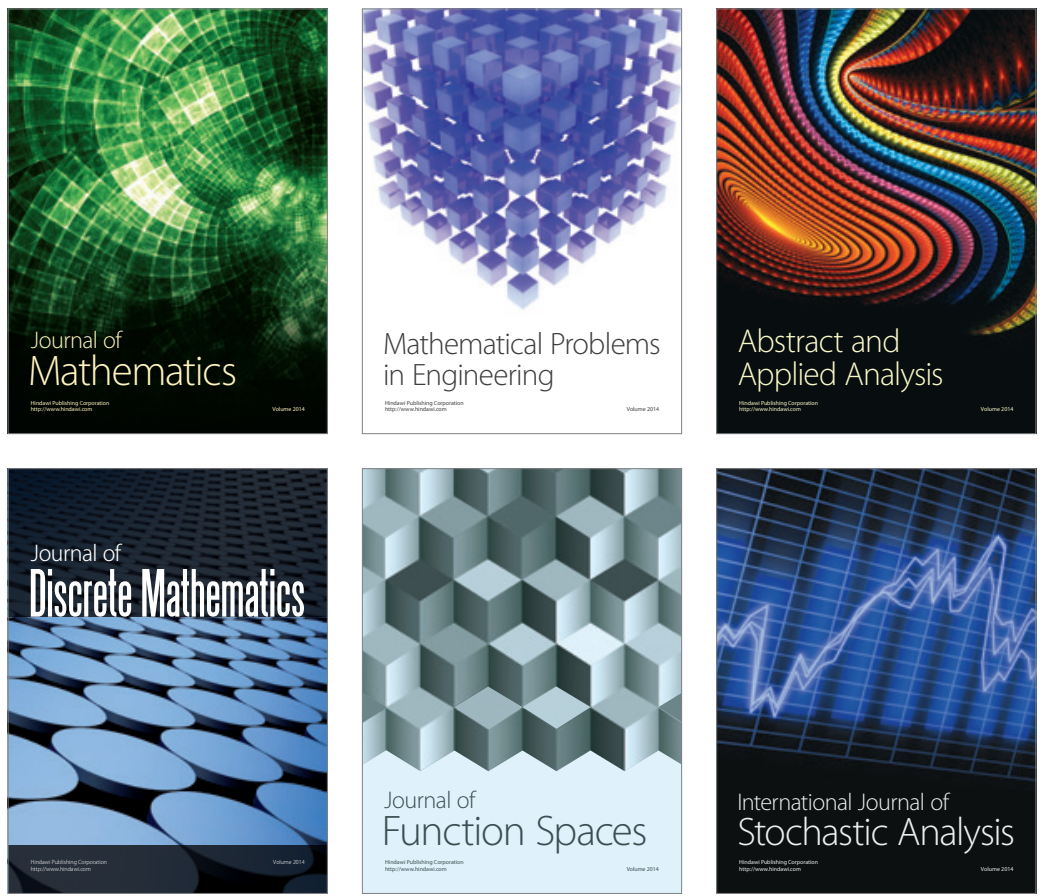

Journal of

Function Spaces

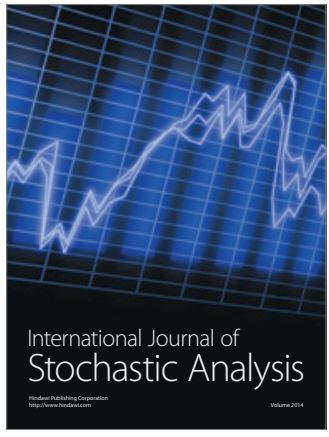

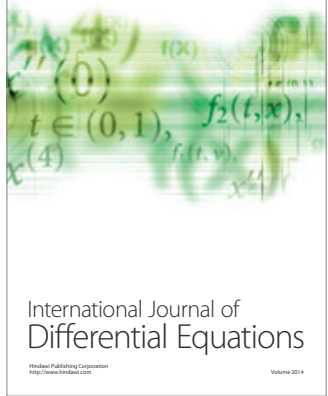
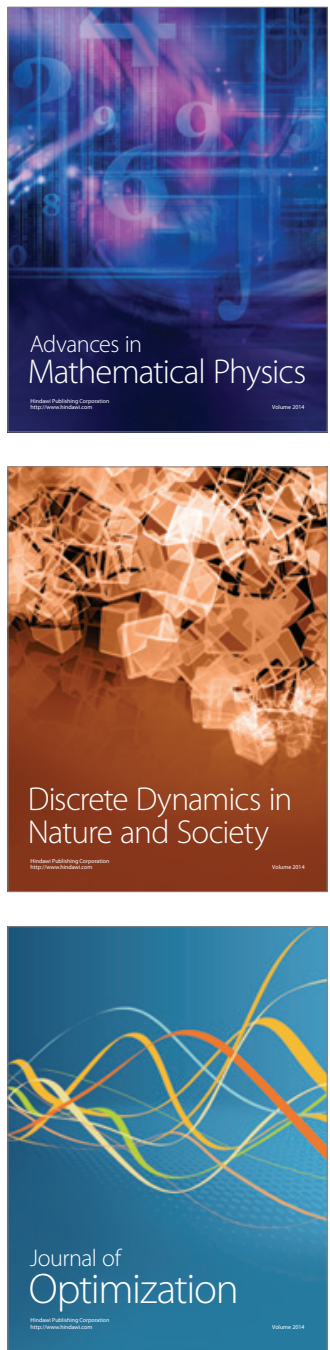